\title{
Moving target detection algorithm: Improved-Vibe
} Yuntao Dou ${ }^{1, a}$, ZhenghuaLiu ${ }^{1,2, b}$, Le Chang $^{1, \text { a }}$, Huang Jianping ${ }^{3, \mathrm{~b}}$

${ }^{1}$ School of Automation Science and Electrical Engineering, Beihang University, Beijing 100191, China

${ }^{2}$ Science and Technology on Aircraft Control Laboratory, Beihang University, Beijing 100191, China

${ }^{3} \mathrm{AVIC}$, China Helicopter Research and Development Institute, Jing Dezhen City, Jiangxi Province, 333001, China

aemail:woshidyt@hotmail.com, bemail:amenia@163.com

\section{Keywords: Background Modeling; Motion Detection; Ghost Processing; Shadow Suppression}

\begin{abstract}
Vibealgorithm initializes the background model very quickly by using the first frame.However, when the target has been in the first frame, the ghostwill be produced with the initialization.And the original Vibe algorithm has no special inhibition about it.In addition, the shadow of the moving target will be detected as a goal, which will influence the accuracy of detection.In order to solve these problems, this paper presents an improved algorithm to inhibit the ghost and shadows.If a pixelis judged as a foreground pixel for many times, it will be compared with the pixel which is the closest one of it, and judged whether they are belong to the same area. Besides, this paper uses a fusion method based on LBP operator and gray space to delete the shadow. Experimental results show that the Improved-Vibe algorithm can absorb the ghost quickly and reduce the influence of shadow compared with the original Vibe algorithm.
\end{abstract}

\section{Introduction}

Moving target detection is one of the important directions of research in the field of computer vision. And it is the foundation of target tracking, behavior recognition, etc.

Moving target detection algorithms can be divided into the following categories: framedifferencemethod[1],opticalflow method[2] and backgroundsubtraction method[3].According to the difference modeling, the background subtraction method includes GMM[4], Code Book[5], Vibe[6], etc.

Frame difference method is easy to design and has a small amount of calculation. But it could make mistakes when the target moves slowly.Opticalflow method has a high accuracy, but its amount of calculation is too large to be applied to the high real-time occasion.Parameter estimation is slow in GMM, so that it is difficult for GMM to meet the real-time requirements. The fixed learning threshold of code book model is easy to lead to unlimited expansion of code.Vibe algorithm can extract the target in the second frame, and has good adaptability and real-time performance. So it is the best choice of detection.

However, the original Vibe algorithm treats the ghost as foreground without any special processing [6]. The ghost has serious influence on detection, and will bring in mistakes in tracking and recognition.One researcher presents a method to detect the ghost by matching the inner and outer edge histogram[7]. And one study uses inter frame difference to restrainghost[8].Based on that the pixel of ghost is able to match with the background model of its neighbor pixel, the literature[9] proposes a new method.On the basis of the above articles, this paper proposes a ghost detection algorithm based on adjacent pixel consistency.

Additionally, the shadow of moving target will be treated as target, leading to false detection.Based on the YUV color space the literature [10] puts forward a suppression method.Literature [11] proposes a method on the basics of gray scale relation.In view of these researches mentioned above, this paper proposes an algorithm based on the fusion operator LBP texture feature and gray scale relation.

With the suppression of ghost and shadow, the robustness and accuracy of the original Vibe 
algorithm are improved very well.

\section{VibeAlgorithm}

Vibe algorithmis proposed by O. Barnichand M. VanDroogenbroeck of Belgium in 2009 for quick background extraction and the moving target detection [6].On the basis ofspatial consistency principle, Vibe algorithm uses the first frame to initialize the background model.It also introduces the randomization into modelingfirst time.

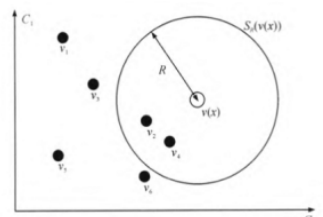

Fig.1. Vibe background model

As shown in fig.1, the first process is to build background model for every pixel which is a collection of $\mathrm{N}$ samples. We can establish a formula:

$\mathrm{M}(\mathrm{x})=\left\{v_{1}, v_{2}, v_{3} \ldots \ldots v_{N}\right\}(1)$

Formally, just like formula (2), for the current pixel values $\mathrm{v}(\mathrm{x})$ of $\mathrm{x}$, let us setting threshold $\mathrm{R}$, and calculating the number of common samples of the $\{v(x)-R, v(x)+R\}$ with $M(x)$.

$\#\{\{\mathrm{v}(\mathrm{x})-\mathrm{R}, \mathrm{v}(\mathrm{x})+\mathrm{R}\} \cap \mathrm{M}(\mathrm{x})\} \quad(2)$

If the result of formula (2) is greater than theset value \#min, the pixel ofx will be treated as a member of foreground, otherwise a member ofbackground.

Usuallyweneed a certain length of video sequence to complete theinitialization. However, only with the first frame, can Vibe initialize the background model.Due to one frame image could not include $\mathrm{N}$ samples of one certain pixel. According to the spatial consistencyprinciple, we fill the background model of one pixel for $\mathrm{N}$ times with its neighbor pixels. These neighbor pixels will be randomly chosen from its eight neighbor pixels.From the above laws we can find:

$\mathrm{M}^{0}(\mathrm{x})=\left\{\mathrm{v}^{0}(\mathrm{y} \mid \mathrm{y} \in \mathrm{NG}(\mathrm{x}))\right\}$ (3)

In the formula (3), 0 represents the initial time, and NG (x) represents the eight neighbor pixels.

Vibe adopts a conservative model updating strategy, and foreground pixels will never be used to fill the background model.

When xis treated as a background pixel, it has a probability of one of the $\oint$ points to be used to update its own background model. For the $\mathrm{N}$ members of $\mathrm{M}(\mathrm{x})$, according a uniform rules, Vibe selects one randomly to replace it with x.At the same time, it also has the probability of one of the $\oint$ points to update the background model of one of its neighbor pixels, which is selected randomly from its eight neighborpixels.

The present time is $t$, in future time $(t+d t)$, the probability of one certain sample is still in the background model is equal to:

$\mathrm{P}(\mathrm{t}, \mathrm{t}+\mathrm{dt})=\mathrm{e}^{-\ln \left(\frac{\mathrm{N}}{\mathrm{N}-1}\right) \mathrm{dt}}$

It shows the decay of the chance for one certain sample to survive is exponential. Via secondary sampling, Vibecan use the limited samples to express infinite.Besides, the transmissions of pixel's valueamong its neighbor makesthe judgment of background has integrity, andweakens the influence of disturbance.

\section{Processing of Shadow and Ghost}

In order to improve robustness and accuracy ofVibe,this paper adoptseffective methods to process ghost and shadow. The algorithmis shown in Fig. 2. 


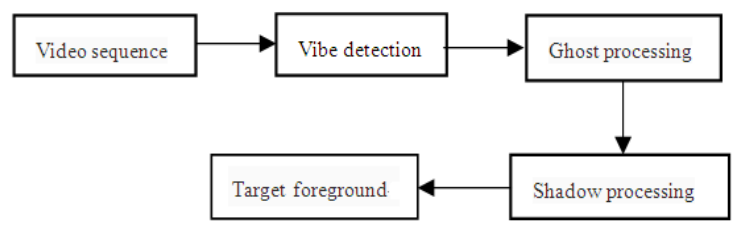

Fig.2.Structure of the algorithm

\section{A. Ghost processing method}

In initialization, Vibe treats the target which appears in the first frame as a part of background. When the target leaves original position, the true background we sampling is not able to match the background model, which results in that the pixel ofbackground will be wrongly detected as a member of foreground. It is the ghost [12].

According to thespatial consistency principle, the values of adjacent pixels are approximately equal at some point.Based on this theory, this paper proposes an improved algorithm as follows:

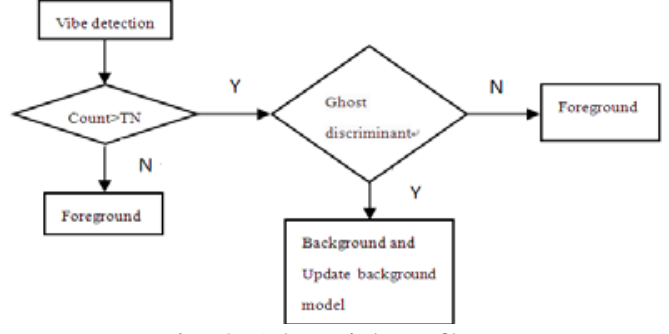

Fig.3.Algorithm flow

The design of algorithm:

As shown above, if somepixel is detected as a foreground pixel for many times continuously, we consider it as an apparentghost pixel.

When we deal with the pixels of one frame, we always scan the frame from top to bottom and from left to right. So that the neighbor pixels we use only include the four pixels marked with $\mathrm{k}$, just like shown in fig.4.

\begin{tabular}{|c|c|c|}
\hline$k$ & $k$ & $k$ \\
\hline$k$ & $x$ & $u$ \\
\hline$u$ & $u$ & $u$ \\
\hline
\end{tabular}

Fig.4.Eightneighbormodel

Based on the rule, we have knownwhether the 4 neighbor points marked with $\mathrm{k}$ belong to ghost or not. Even if one belongs to ghost, we also have dealt with it. Therefore, they are trustworthy when we deal with $x$.In addition, the ghost is big and may contain the 4 unknown neighbor points marked with u.Sothese unknown points cannot be used as trustworthy template. If pixel x isdetected asa ghost pixel, we should let it be trustworthy. And with a certain probability, we replace the background model of $\mathrm{x}$ and its neighbor pixels' background model with pixel x.Then we can suppress ghost fromoutside to inside, and eliminate the ghost quickly.

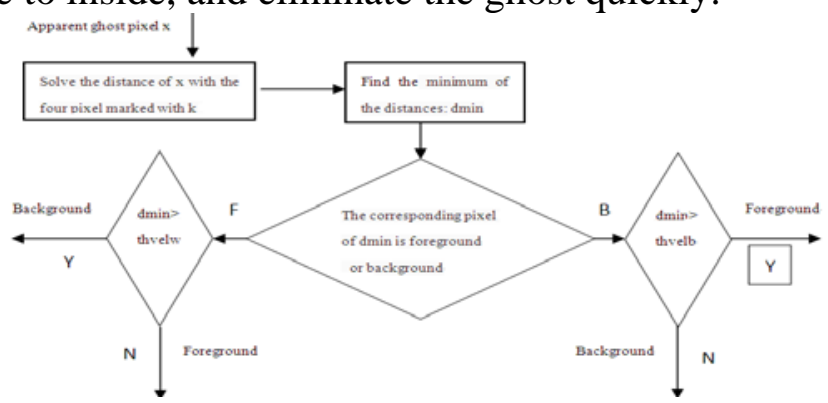

Fig.5.Ghost processing algorithm

Detecting and updating process is shown as follows:

As shown in fig.4, we solve the absolute value of the four differences of the value pixel $\mathrm{x}$ minus 
the pixel $k$, and find the minimum value of result using the following formula.

$\mathrm{dmin}=\min \{\mathrm{d} 1, \mathrm{~d} 2, \mathrm{~d} 3, \mathrm{~d} 4\}(5)$

If dminis in the threshold range, we canconclude that the pixel corresponding to $x$ matches the pixel corresponding to dmin.

When pixel corresponding to dmin belongstoforeground, if $\mathrm{dmin}>$ thvelw, the pixel $\mathrm{x}$ belongs tobackground, otherwise foreground. Whenpixel corresponding to dmin belongs to background, if $\mathrm{dmin}>$ thvelb, the pixel $\mathrm{x}$ belongs to foreground, otherwise background.

If $\mathrm{x}$ is assumed to be ghost pixel, with a certain probability, we replace the sample ofits background model and the sample of its neighbor pixels' the background model of with $\mathrm{x}$.

With the twice model updating process, the algorithm can rapidly suppress ghost, and ensure the purity of the model samples.

\section{B. Shadow processing method}

Normally, shadows cannot affect detection, for instance, the shadows of stock-stilltarget and micro target (leaves shaking etc.).But the shadow of the moving target will be detected aspart of the detection result.

Based on the shadow attributes, when a pixel is in shadow, it is a linear relationship between its current gray value and its original gray value described as formula(6).

$$
\mathrm{p}(\mathrm{i}, \mathrm{j})=\frac{B(i, j)}{O(i, j)} \quad(0.2<p<0.8)(6)
$$

The original gray value is $O(i, j)$ and $B(i, j)$ is the gray value covered with shadow. In the foreground, if a pixel's gray value and its initial gray value satisfy formula (6), we can conclude the pixel is in shadow, and delete it.

However, the experimental results show that when the gray value of moving target is close to background, this method will leadparts of target to be treated as shadowand be suppressed.

For solvingthis problem, this paper introduces LBP texture feature asconstraint condition. Only when one certain pixel satisfies LBP constraint condition and the formula (6) at the same time, will it be treat as a part of shadow.

In fig.6, the LBP operator is defined in a $3 * 3$ window with the window center pixel as the threshold.

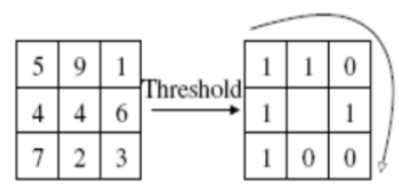

Fig.6. LBP operator

Comparing the gray value of the adjacent 8 pixels with the center one, if one adjacent pixel is bigger than the center one, its place will be marked as 1, otherwise as 0 .In this way, the 8 points of the $3 * 3$ window can generate an 8 bit binary number which is the LBP feature vector of the center pixel. We can use the vector to reflect the texture information of the region.

Researches show shadow will notaltered LBP feature vector of one pixelseverely. Therefore, via comparing the original LBP feature vector with the current LBP feature vector we can find the true shadow.

$$
\begin{aligned}
& \mathrm{N}=\operatorname{num}(\overrightarrow{o L B P}-\overrightarrow{b L B P}) \\
& \mathrm{N}<N_{\max }
\end{aligned}
$$

In formula (7), $\overrightarrow{o L B P}$ represents the original LBP feature vector, and $\overrightarrow{\mathrm{bLBP}}$ represents the currentLBP feature vector.

Formula (8) means the total number of different values between the two vectors. As shown in formula(8), ifNis less than $N_{\max }$, then we can conclude this pixel is in shadow.

In summary, the algorithm flow this paper designs is shown in fig.7: 


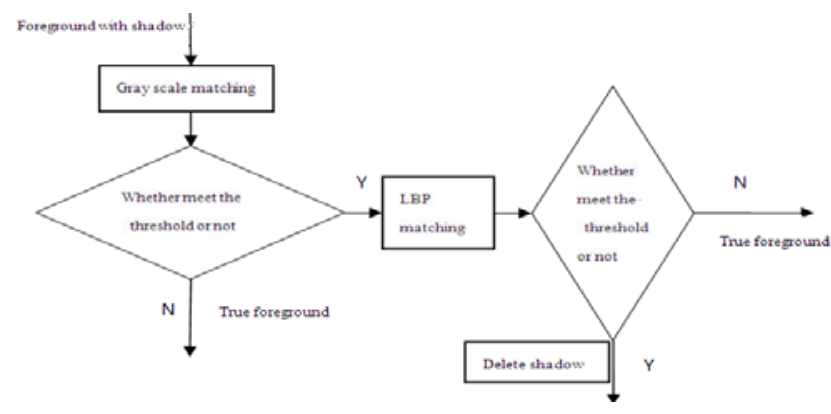

Fig.7. Shadow suppression algorithm

When one pixel satisfies the twoconditions at the same time, it is in shadow and can be deleted. After this process, we can get the true target.

\section{The results of experiment}

In this paper, the experimental environment is VS2008 with the configuration of the opencv2.1. We use the Lenovo YangtianM2620D as our hardware platform andits single-coreprocessor is Intel Celeron E3400 2.6GHz. Besides, the operating system is Windows XP 5.1. And the video this paper uses is commonly usedin visual domain whoseresolution is $320 \times 240$ and frame rate is $24 \mathrm{pfs}$.

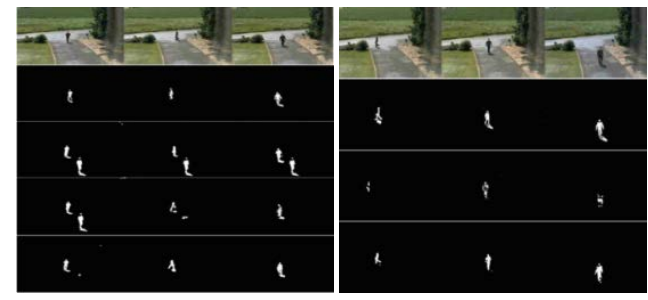

(a) ghostprocessing(b) shadow processing

Fig.8. Experimental results

As shown in Fig.8.a, these pictures(left to right)are $33^{\text {rd }}, 117^{\text {th }}$ and $333^{\text {rd }}$ frame, and (topto bottom)are Input-image, Ground-truth, Vibe, the algorithm of literature [9] and the algorithm of this paper. The result shows the algorithm of this paper can eliminate ghost more quickly.

As shown in Fig.8.b, these pictures(left to right)are $132^{\text {nd }}, 296^{\text {th }}$ and $303^{\text {rd }}$ frame, and (top to bottom) are Input-image, Ground-truth, thealgorithm of literature [11] and the algorithm of this paper. The result shows that the effect of this paper's algorithm is better than before.

\begin{tabular}{r|c|c|c|c|c} 
& \multicolumn{5}{c}{ Table1. Performance of the algorithms } \\
\cline { 1 - 1 } & Vibe & Lit.9 & Lit.11 & T-paper \\
\hline S.pr & & $125^{\text {th }}$ & & $34^{\text {th }}$ & Notes: \\
Rate & 21.6 & & $\pm 43 \%$ & $\pm 12 \%$ & $\begin{array}{c}\text { thvelw }=24 \\
\text { thvelb=27 }\end{array}$
\end{tabular}

G.pr: ghost process; S.pr: shadow process;Lit.9: the algorithm of literature [9];

Lit.11: the algorithm of literature [11];T-Paper: the algorithm of this paper;

Description:

It need about 125 frames to eliminate ghost completely by the algorithm of literature [9]; and the algorithm of this paper need about 33 frames.The error range is about $\pm 43 \%$ when using the algorithm of literature [11], and The error range is about $\pm 12 \%$ when using the algorithm of this paper.Rate means the average number of framesevery second.

\section{Conclusions}

Since the Vibe has no special treatment for ghost, and this will lead the ghost cannot be inhibited quickly and effectively.Besides, the shadows also have some negative influence on the detection and subsequent tracking and counting.In order to solve these problems mentioned above, this paper proposes the Improved-Vibe algorithm with suppression of ghost and shadow. 
Experiments show that the Improved-Vibe algorithm can eliminate ghost quickly and suppressshadow effectively.Although the algorithm of this paper contains the suppression of ghostand shadow, as shown in Table1, this algorithmcan still be able to meet real-time requirements.

\section{ACKNOWLEDGEMENTS}

In this paper, this work was partly supported by the grants of National Natural Science Foundation of China(No. 61305132) and Aeronautical Science Foundation of China(No. 20135751040).

\section{References}

[1]JianZhao,TheResearch of Moving Target Detection Method Based on Three-frame Difference[D],Xi'an,Xidian University,2013.

[2]Horn B $\quad \mathrm{K} \quad$ P,Schunk $\mathrm{B} \quad$ G.Determining optical flow[J].Artificial Intelligence.198117(123)185-203.

[3]J. Davis and V. Sharma, Background-subtraction in thermal imagery using contour saliency[C].IEEE International Journal of Computer Vision.2007 (77) 161-181

[4]D. Lee. Effectivegaussianmixture learning for video background subtraction[C].IEEE Transactions on Pattern Analysis and Machine Intelligence. 2005 (27) 827-832.

[5]CaixiongChenand so on.Detection algorithm based on block modeandcodebook model[J].Journal of China University of Metrology.201323(2)125-130.

[6]O.Barnich and M.Van Droogenbroeck. ViBe: A universal background subtraction algorithm for video sequences[J].IEEE Transactions on Image Processing.201120(6)1709-1724.

[7]Liang Hua, Moving object detection and tracking for multi-camera video surveillance [D].Changsha: National University of Defense Technology, 2009.

[8]Li Y, Chen W, Jiang R. The Integration Adjacent Frame Difference of Improved ViBe for Foreground Object Detection[C]. Wireless Communications, Networking and Mobile Computing (WiCOM), 20117th International Conference on.IEEE, 2011.

[9] Liang Chen, Xiaozhu Chenand so on. Ghost suppression algorithm based on ViBe[J]. Journal of China University of Metrology.201324 (4)425-429.

[10]YeYuand so on.EVibe: An improved Vibe algorithm for detecting moving objects[J]. Chinese Journal of Scientific Instrument. 201435 (4)924-931.

[11]Qin Lin. Research on Moving object Detection and Shadow Suppression[D],Zhen Jiang, Jiangsu University,2010.

[12]R.Cucchiara, C. Granaand so on. Detecting moving objects, ghosts, and shadows in video streams[J]. IEEE Transactions on Pattern Analysis and Machine Intelligence.2003 (25) 1337-1342. 